\section{Helicobacter pylori clarithromycin resistance assessment: are gastric antral biopsies sufficient?}

\author{
Vincenzo De Francesco, ${ }^{1}$ \\ Floriana Giorgio, ${ }^{1}$ Enzo lerardi, ${ }^{1}$ \\ Cristina D'Ercole, ${ }^{2}$ Cesare Hassan, ${ }^{2}$ \\ Angelo Zullo2 \\ 1Section of Gastroenterology, \\ Department of Medical Sciences, \\ University of Foggia, Foggia; \\ ${ }^{2}$ Gastroenterology and Digestive \\ Endoscopy, Nuovo Regina Margherita \\ Hospital, Rome, Italy
}

very high accuracy (98\%), ${ }^{3,4}$ these techniques allow to assess a singular condition, i.e. the heteroresistance. ${ }^{5}$ Such a status describes the coexistence of resistant and susceptible bacterial strains towards to a specific antibiotic in the same patient. Heteroresistance may be either intraniche (coexistent susceptible and resistant strains in the same gastric site) or interniche (separate susceptible and resistant strains in different gastric site, i.e. antrum and gastric body). Based on the latter possibility, antibiotic assessment by using in only antral biopsies - as usually performed for bacterial culture - may underestimate the actual $H$. pylori resistance status. ${ }^{6,7}$ This study aimed to assess primary clarithromycin resistance in $H$. pylori strains separately in antral and gastric body mucosa biopsies.

\section{Abstract}

Gastric biopsy sampling could affect accuracy of Helicobacter pylori clarithromycin resistance assessment due to coexistence of susceptible and resistant strains (i.e. heteroresistance) either in same gastric site (intraniche) or in two different gastric sites (interniche). This study aimed to assess differences in the $H$. pylori clarithromycin resistance prevalence in relation to the gastric biopsy sampling by using Taqman-real time polymerase chain reaction (PCR). The study enrolled 137 patients. Primary clarithromycin resistance was observed in 15 isolates exclusively in antrum, in 7 cases exclusively in gastric body, and in 3 patients in both gastric sites. The overall prevalence of clarithromycin resistance was $13.1 \%$ by using exclusively antral biopsies, and $18.2 \%$ by using biopsies from both gastric sites. Moreover, intra-niche heteroresistance was observed in $19(76 \%)$ out of 25 patients harbouring resistant strains. Our data found a heterogeneous distribution of resistant $H$. pylori strains in the stomach. Similarly to culture, gastric biopsies from both antrum and gastric body are needed to increase the accuracy of PCR-based methods for clarithromycin resistance assessment.

\section{Introduction}

Primary clarithromycin resistance is widely recognized as the main factor reducing efficacy of eradication therapies. ${ }^{1}$ Unfortunately, it is difficult to culture Helicobacter pylori from gastric biopsies. ${ }^{2}$ Therefore, different polymerase chain reaction (PCR)-based techniques have been introduced to overcome some limits of bacterial culture for clarithromycin resistance assessment. Besides a

\section{Case Report}

\section{Patients}

The study enrolled consecutive, $>18$ years old patients never previously treated for $H$. pylori infection who underwent upper endoscopy due to dyspeptic symptoms. All patients underwent endoscopy with biopsies, including 2 specimens from the antrum and 2 from the gastric body. All patients gave informed consent to participate into the study.

\section{TaqMan real time polymerase chain reaction}

DNAs were extracted by using the NucleoSpinnTissue (Macherey-Nagel GmbH\&Co, Germany) according to manufacturer's instructions, applied on embedded-paraffined sections $(10 \mu)$ which constitutes a reliable substrate for DNA analysis likewise fresh material. $^{8}$ The $\mathrm{A} 2142 \mathrm{C}, \mathrm{A} 2142 \mathrm{G}$ and $\mathrm{A} 2143 \mathrm{G}$ point mutations in the 23S rRNA involved in $H$. pylori clarithromycin resistance were detected by molecular analysis after DNA extraction by using a TaqMan real-time PCR, as previously described and validated. ${ }^{8}$ Biopsies from antral were analyzed separately from those of gastric body mucosa.

\section{Results}

The study enrolled 137 patients (Mean age: 48 \pm 12 ; M/F: 54/83) with non-ulcer dyspepsia. Primary clarithromycin resistance was detected exclusively in gastric antrum in 15 isolates (13 with A2143G a 2 with A2142G point mutation), exclusively in gastric body in 7 patients (6 A2143G and 1 with A2142G point mutation), and in both sites in 3 cases (2 A2143G, 1 A2142G). The inter-niche heteroresistance was found in $22(16 \% ; 95 \% \mathrm{CI}=9.9-22.2)$ out of
Correspondence: Dr. Angelo Zullo, Gastroenterology and Digestive Endoscopy, Nuovo Regina Margherita Hospital, via E. Morosini 30, 00153 Rome, Italy

Tel. +39.06.58446608 - Fax: +39.06.58446533.

E-mail: zullo66@yahoo.it

Key words: Helicobacter pylori, clarithromycin resistance, polymerase chain reaction.

Received for publication: 15 October 2011. Revision received: 22 February 2012

This work is licensed under a Creative Commons Attribution NonCommercial 3.0 License (CC BYNC 3.0).

(C) Copyright V. De Francesco et al., 2012

Licensee PAGEPress, Italy

Gastroenterology Insights 2012; 4:e2

doi:10.4081/gi.2012.e2

137 patients, including 15 patients with resistant strains in antrum and susceptible strains in gastric body, and 7 patients with the reverse condition. By using exclusively biopsies from antral mucosa primary clarithromycin resistance was detected in 18 (13.1\%; 95\% CI: 7.418.7) out of 137 patients. Such a value increases to $18.2 \%$ (95\% CI: $11.7-24.7)$ by adding gastric body biopsies assessment. As far as intraniche heteroresistance is regarded, a heterogeneous bacterial population was observed in $9(60 \%)$ out of 15 in gastric antrum, in all 7 (100\%) patients in gastric body, and in all 3 (100\%) with bacterial resistance detected in both gastric sites. Consequently, the intraniche heteroresistance was detected 19 (76\%; 95\% CI: 59.2-92.7) out of 25 patients harbouring resistant strains, with a overall prevalence of heteroresistance status of $13.8 \%$. Finally, the A2143G was the most prevalent point mutation, being present in 21 (84\%; 95\% CI: 69.6-98.3) out of 25 resistant strains, followed by the $\mathrm{A} 2142 \mathrm{G}$ point mutation present in the remaining 4 isolates.

\section{Discussion}

Novel bio-molecular PCR-based tools are able to overcome some limits of bacterial culture in assessing $H$. pylori clarithromycin resistance $^{3,4}$ In detail, PCR-based methods allow to identify even minimal traces of resistant strains among a large susceptible bacterial population (heteroresistance). ${ }^{5}$ This peculiar ability could explain the discrepancies in the clarithromycin resistance rates between culture and PCR-based methods and frequent therapeutic failures in presence of clarithromycin susceptibility at the culture. ${ }^{9}$ The 
need of multiple gastric biopsies taken in different gastric sites as been pointed out for bacterial culture, ${ }^{10}$ but the biopsy sampling for PCR-based methods to assess $H$. pylori resistance has been poorly investigated. Our data found that clarithromycin resistant strains were localized exclusively in gastric body in a relevant number of patients. Therefore, by taking only antral biopsies for antibiotic susceptibility testing the actual resistance status would be underestimated. Our finding confirms in adults the results observed in a paediatric population. ${ }^{11}$ In agreement with previous investigations, ${ }^{4,6,7}$ our results found a high prevalence of heteroresistance for clarithromycin in each gastric sites (intraniche heteroresistance). Moreover, the finding of a significant interniche heteroresistance rate (16\%) would appears of clinical relevance because of the quite constant involvement of the A2143G mutate genotype. Indeed, such a point mutation has been reported to be strongly associated with a therapeutic failure. ${ }^{12-14}$ In conclusion, our data found that by adding antibiotic susceptibility testing on gastric body mucosa specimens increases $H$. pylori clarithromycin resistant rate. Moreover, intragastric distribution of $H$. pylori resistant strains are a highly heterogeneous, both intraniche and interniche.

\section{References}

1. Graham DY, Fischbach L. Helicobacter pylori treatment in the era of increasing antibiotic resistance. Gut 2010;59:1143-53.

2. Zullo A, Hassan C, Lorenzetti R, et al. A clinical practice viewpoint: to culture or not to culture Helicobacter pylori? Dig Liver Dis 2003;35:357-61.

3. van Doorn LJ, Glupczynski Y, Kusters JG, et al. Accurate prediction of macrolide resistance in Helicobacter pylori by a PCR line probe assay for detection of mutations in the 23S rRNA gene: multicenter validation study. Antimicrob Agents Chemother 2001;45:1500-4.

4. Oleastro M, Ménard A, Santos A, et al. Real-time PCR assay for rapid and accurate detection of point mutations conferring resistance to clarithromycin in Helicobacter pylori. J Clin Microbiol 2003;4:397-402.

5. Vega AE, Alarcón T, Domingo D, LópezBrea M. Detection of clarithromycinresistant Helicobacter pylori in frozen gastric biopsies from pediatric patients by a commercially available fluorescent in situ hybridization. Diagn Microbiol Infect Dis 2007;59:421-3.

6. Matsuoka M, Yoshida Y, Hayakawa K, et al. Simultaneous colonisation of Helicobacter pylori with and without mutations in the 23S rRNA gene in patients with no history of clarithromycin exposure. Gut 1999;45: 503-7.

7. Kim JJ, Kim JG, Kwon DH. Mixed-infection of antibiotic susceptible and resistant Helicobacter pylori isolates in a single patient and underestimation of antimicrobial susceptibility testing. Helicobacter 2003;8:202-6.
8. De Francesco V, Margiotta M, Zullo A, et al. Primary clarithromycin resistance in Italy assessed on Helicobacter pylori DNA sequences by TaqMan real-time polymerase chain reaction. Aliment Pharmacol Ther 2006;23:429-35.

9. De Francesco V, Zullo A, Ierardi E, et al. Phenotypic and genotypic Helicobacter pylori clarithromycin resistance and therapeutic outcome: benefits and limits. J Antimicrob Chemother 2010;65:327-32.

10. Masuda H, Hiyama T, Yoshihara M, et al. Necessity of multiple gastric biopsies from different sites for detection of clarithromycin-resistant Helicobacter pylori strains. Scand J Gastroenterol. 2003;38: 942-6.

11. Caristo E, Parola A, Rapa A, et al. Clarithromycin resistance of Helicobacter pylori strains isolated from children' gastric antrum and fundus as assessed by fluorescent in-situ hybridization and culture on four-sector agar plates. Helicobacter. 2008; 13: 557-63

12. Stone GG, Shortridge D, Versalovic J, et al. A PCR-oligonucleotide ligation assay to determine the prevalence of $23 \mathrm{~S}$ rRNA gene mutations in clarithromycin-resistant Helicobacter pylori. Antimicrob Agents Chemother 1997;41:712-4.

13. Owen RJ. Molecular testing for antibiotic resistance in Helicobacter pylori. Gut 2002;50:285-9.

14. De Francesco V, Margiotta M, Zullo A, et al. Clarithromycin-resistant genotypes and eradication of Helicobacter pylori. Ann Int Med 2006;144:94-100. 